\title{
The effect of medial open wedge high tibial osteotomy on the patellofemoral joint: comparative analysis according to the preexisting cartilage status
}

Hyun-Soo Moon 1,2, Chong-Hyuk Choi ${ }^{1,3}$, Min Jung ${ }^{1,3}$, Sang-Hoon Park ${ }^{4}$, Dae-Young Lee ${ }^{1,5}$, Jong-Kwan Shin ${ }^{3}$ and Sung-Hwan $\operatorname{Kim}^{1,2^{*}}$

\begin{abstract}
Background: Although it has been known that medial open wedge high tibial osteotomy (MOWHTO) would adversely affect the patellofemoral joint, no previous study examined the surgical outcome of MOWHTO according to the preexisting cartilage status of the patellofemoral joint. The aim of this study was to investigate the effect of MOWHTO on the patellofemoral joint with regard to objective and subjective aspects according to the preexisting cartilage status.
\end{abstract}

Methods: Ninety-two patients who underwent MOWHTO and a following second-look arthroscopic assessment were included in this study. The patients were divided into two groups according to the preexisting cartilage status of the patellofemoral joint: group 1 (International Cartilage Repair Society [ICRS] grade 2 or 3 ) and group 2 (ICRS grade 0 or 1). Comparative analysis was performed regarding clinical scores, radiographic parameters, and arthroscopic measurements between the two groups.

Results: Clinical outcomes showed overall improvement from baseline to the time of second-look operation, with no significant difference between the two groups at each time point. There were no significant differences in radiographic parameters between the two groups. Radiographic grade of patellofemoral osteoarthritis in both groups showed a tendency to progress, without statistical significance. In arthroscopic assessment, the size of the cartilage lesion on the patellofemoral joint increased with time in both groups $(P=0.003)$, but the degree of change over time between the two groups was not statistically significant. Consistently, there was no significant difference in the frequency of progression of cartilage lesion grade in the patellofemoral joint between the two groups.

Conclusions: MOWHTO would contribute to osteoarthritis progression of the patellofemoral joint regardless of the preexisting cartilage status, without an association with clinical outcomes in short-term follow-up.

Keywords: Knee, High tibial osteotomy, Patellofemoral joint, Cartilage lesion

\footnotetext{
* Correspondence: orthohwan@gmail.com; orthohwan@yuhs.ac

${ }^{1}$ Arthroscopy and Joint Research Institute, Yonsei University College of

Medicine, Seoul, Republic of Korea

${ }^{2}$ Department of Orthopedic Surgery, Gangnam Severance Hospital, Yonsei

University College of Medicine, Seoul, Republic of Korea

Full list of author information is available at the end of the article
}

(c) The Author(s). 2019 Open Access This article is distributed under the terms of the Creative Commons Attribution 4.0 International License (http://creativecommons.org/licenses/by/4.0/) which permits unrestricted use, distribution, and reproduction in any medium, provided you give appropriate credit to the original author(s) and the source, provide a link to the Creative Commons license, and indicate if changes were made. The Creative Commons Public Domain Dedication waiver (http://creativecommons.org/publicdomain/zero/1.0/) applies to the data made available in this article, unless otherwise stated. 


\section{Background}

Medial open wedge high tibial osteotomy (MOWHTO) is an effective surgical procedure for the treatment of medial compartment osteoarthritis of the knee as well as for the correction of lower extremity malalignment [1-3]. With favorable clinical outcomes and improved surgical techniques, MOWHTO has become increasingly popular [4-6].

Although numerous advantages of MOWHTO have been addressed, it has been reported that MOWHTO would adversely affect the patellofemoral joint. Several studies state that MOWHTO leads to patella baja, subsequently causing increased patellofemoral contact pressure [7-9]. Varusvalgus alignment was also reported to affect the progression of patellofemoral osteoarthritis in a compartment-specific manner [10]. Recently, several studies investigating the effect of MOWHTO on the patellofemoral joint using an arthroscopic assessment also reported overall deterioration of the articular cartilage of the patellofemoral joint over time as a result of MOWHTO [11-14].

However, it is difficult to conclude that MOWHTO definitely contributes to the deterioration of the articular cartilage of the patellofemoral joint. There are various factors that could affect the progression of the patellofemoral osteoarthritis [15]. In particular, the possibility of the normal progression of preexisting cartilage lesions could not be ruled out. Focal cartilage lesions of the joints are known to cause stress concentration in the rim of the defect, acting as a leading factor of arthritis [16]. Due to the nature of the study design of the abovementioned studies [11-14], which assessed articular cartilage status arthroscopically, could not provide details according to the presence or absence of preexisting articular cartilage lesions. To determine the contribution of MOWHTO to the progression of patellofemoral joint osteoarthritis, the effect of preexisting cartilage lesions should be investigated. To the best of the authors' knowledge, no previous study examined the outcome of MOWHTO according to the preexisting cartilage status of the patellofemoral joint.

The purpose of this study was to investigate the effect of MOWHTO on the patellofemoral joint with regard to the objective and subjective aspects according to the preexisting cartilage status. The hypothesis was as follows: (1) MOWHTO would contribute to progression of patellofemoral joint osteoarthritis regardless of the presence of preexisting cartilage lesions in the objective perspective and (2) clinical outcome of MOWHTO in patients with preexisting cartilage lesion of patellofemoral joint would not be inferior compared to those with normal patellofemoral joint cartilage in the subjective perspective.

\section{Methods}

This study was approved by the institutional review board prior to the study, which waived the requirement for informed consent from patients owing to the retrospective nature of the study. Data of 178 consecutive patients, who underwent biplane MOWHTO by a single orthopedic surgeon in a single institution between January 2010 and February 2018 were reviewed retrospectively. Of those, patients who underwent second-look arthroscopic assessment were eligible to be included in this study. The exclusion criteria were as follows: (1) history of previous surgical treatment of the knee, (2) surgical site infection, (3) additional surgical procedure of the same knee during the follow-up period, and (4) the same subsequent surgical procedure of the opposite knee during the follow-up period. In addition, the patients with a time more than 3 years from MOWHTO to second-look operation, and the patients who underwent marrow stimulation procedure on the International Cartilage Repair Society (ICRS) grade 4 cartilage lesion in patellofemoral joint at initial operation were excluded to increase comparability. As a result, a total of 92 cases who met abovementioned conditions were included in this study and divided into 2 groups according to the preexisting cartilage status of the patellofemoral joint. The distribution of patients was as follows: (1) group 1, 59 patients with patellofemoral joint cartilage lesion greater than ICRS grade 2 and (2) group 2, 33 patients with cartilage lesion of ICRS grade 0 or 1 (Fig. 1). For the cartilage lesions in both the patella and trochlea, higher grade lesions were used as a reference. Baseline characteristics were similar between the two groups (Table 1). The mean time from MOWHTO to the second-look operation was $21.7 \pm 6.2$ and $21.2 \pm 5.3$ months for group 1 and 2, respectively. In addition, subgroup analysis of group 1 , revealed that there were no statistically significant within-group differences (Additional file 1).

\section{Surgical indications and procedure}

Surgical indications for MOWHTO were as follows: (1) patients younger than 65 years who had medial compartment osteoarthritis with varus malalignment, (2) activity-related medial-sided knee pain, (3) good range of motion (arc of motion $>100^{\circ}$ and flexion contracture $<15^{\circ}$ ) and without joint instability. MOWHTO was not indicated for the patients who complained of anterior knee pain associated with activities, such as squatting and stair climbing or descending. Moreover, patients with more than stage 3 of patellofemoral osteoarthritis according to Iwano's osteoarthritis classification system were excluded regardless of symptoms [17]. All patients were recommended to perform hardware removal if bony consolidation was confirmed, due to the possible postoperative pain owing to local irritation of the plate. If the plate removal was planned, secondlook arthroscopic assessment was recommended to be performed at the same time.

In all patients, preoperative surgical planning for obtaining appropriate alignment of the lower limb was performed according to the Miniaci method [18], realigning the mechanical axis to be located at the Fujisawa 
178 Patients who underwent MOWHTO (January 2010 to February 2018)

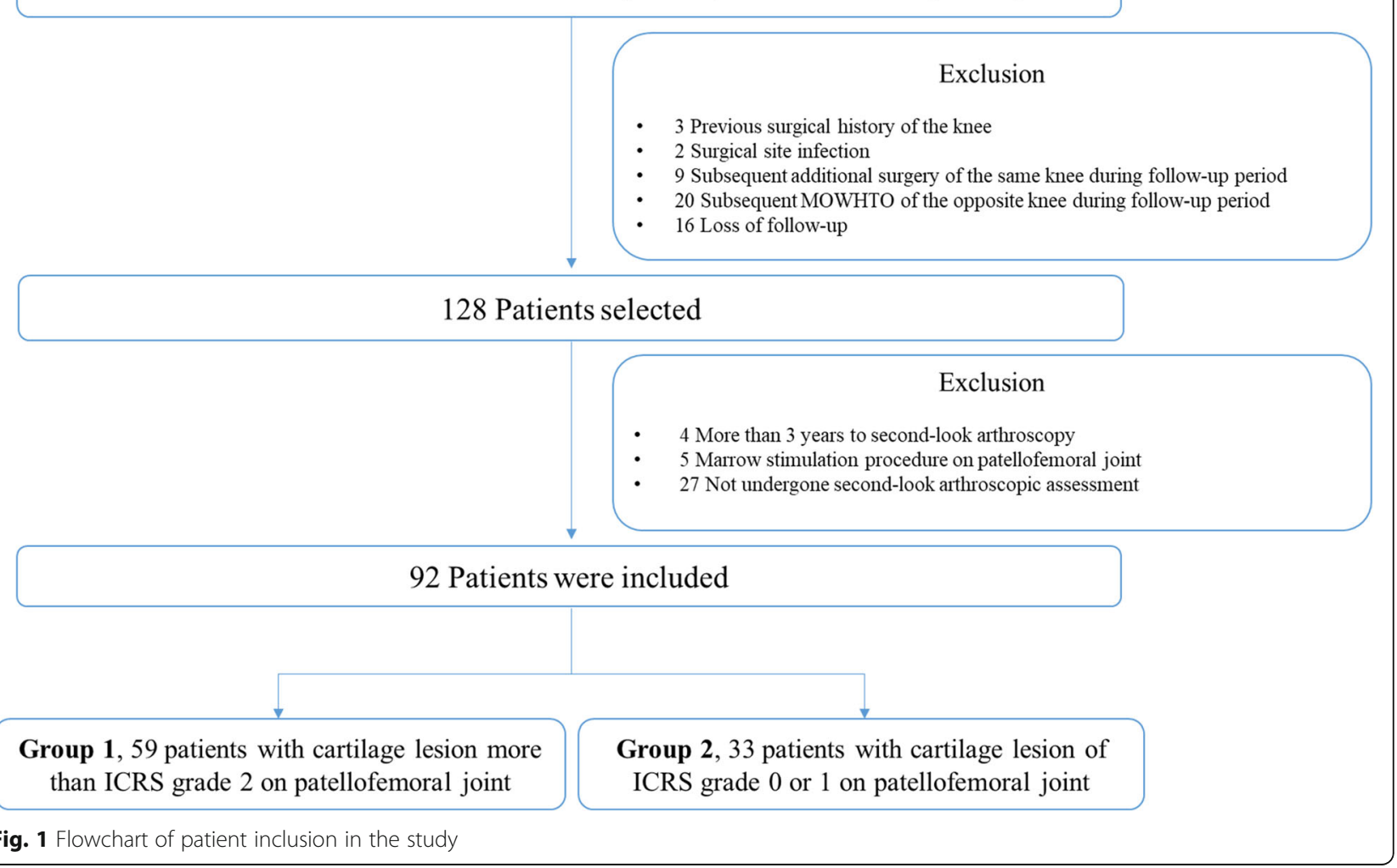

point [19]. Prior to the osteotomy procedure, diagnostic arthroscopy was performed, and the status of articular cartilage was evaluated thoroughly. Cartilage procedure such as debridement and chondroplasty was not performed on the cartilage lesion of patellofemoral joint. After the arthroscopic assessment, biplane MOWHTO was performed. To expose the medial proximal tibia, an approximately $6-8-\mathrm{cm}$ oblique skin incision was made from $1 \mathrm{~cm}$ below the joint line to the pes anserinus tendons between the tibial tuberosity and the inner border of the tibia. Then, the distal superficial medial collateral ligament was released and underlying periosteum was removed. Two starting guide wires for transverse osteotomy were inserted parallel from the upper border of the pes anserinus tendons toward the upper portion of the fibular head. Prior to transverse osteotomy, the separate oblique vertical osteotomy in the coronal plane was made $1 \mathrm{~cm}$ behind the tibial tuberosity. Transverse osteotomy was initiated subsequently with an oscillating saw along the two guide wires leaving the lateral most at $1 \mathrm{~cm}$ of proximal tibia as a hinge. Osteotomy site was opened gradually using several chisels and a spreader device. After the desired correction was achieved, TomoFix plate (Synthes, West Chester, PA) was applied and fixed to the medial proximal tibia over the osteotomy site.
Postoperatively, patients were instructed to begin crutch-assisted progressive weight-bearing ambulation as tolerated. After restricting the knee range motion for 2 weeks with splint immobilization, exercise for knee range motion was initiated with hinged knee brace. Six weeks after the surgery, all patients were encouraged to remove both crutch and hinged knee brace.

\section{Evaluation}

Comparative analysis of clinical outcomes, radiographic factors, and arthroscopic measurements were performed. Clinical outcomes were assessed preoperatively and at the time of second-look operation using various patientreported knee rating scales, including the visual analog scale (VAS) [20], International Knee Documentation Committee (IKDC) subjective score [21], and Kujala scale (anterior knee pain scale) [22]. The degree of osteoarthritis was radiographically assessed using the Kellgren-Lawrence grading system for the tibiofemoral joint and the Iwano classification system for the patellofemoral joint [17, 23]. Various radiographic parameters possibly associated with the preexisting condition of the patellofemoral joint, including load-bearing axis deviation [24], hip-knee-ankle angle [24], medial proximal tibial angle [25], joint line convergence angle [25], posterior tibial slope [26], and the presence of trochlear 
Table 1 Comparison of baseline characteristics

\begin{tabular}{|c|c|c|c|}
\hline Variable & $\begin{array}{l}\text { Group } 1 \\
(n=59)\end{array}$ & $\begin{array}{l}\text { Group } 2 \\
(n=33)\end{array}$ & $P$ value \\
\hline Age $^{a}$ (year) & $54.3 \pm 5.2$ & $55.5 \pm 4.4$ & 0.261 \\
\hline Gender b & & & 0.745 \\
\hline Male & $16(27.1 \%)$ & $10(30.3 \%)$ & \\
\hline Female & $43(72.9 \%)$ & $23(69.7 \%)$ & \\
\hline $\mathrm{BMI}^{\mathrm{a}}(\mathrm{kg} / \mathrm{m} 2)$ & $26.2 \pm 5.9$ & $25.6 \pm 5.2$ & 0.613 \\
\hline Load-bearing axis deviation ${ }^{a}(\%)$ & $19.0 \pm 11.7$ & $19.3 \pm 10.5$ & 0.905 \\
\hline Hip-Knee-Ankle angle ${ }^{a}\left(\right.$ varus $\left.^{\circ}\right)$ & $7.9 \pm 2.9$ & $7.7 \pm 2.7$ & 0.762 \\
\hline Medial proximal tibial angle ${ }^{a}\left(^{\circ}\right)$ & $84.0 \pm 2.2$ & $84.1 \pm 1.9$ & 0.843 \\
\hline Joint line convergence angle ${ }^{a}\left({ }^{\circ}\right)$ & $3.2 \pm 1.7$ & $2.9 \pm 1.7$ & 0.437 \\
\hline Posterior tibial slope $\left({ }^{\circ}\right)$ & $5.7 \pm 3.2$ & $6.2 \pm 4.0$ & 0.590 \\
\hline Blackburne-Peel ratio ${ }^{a}$ & $0.9 \pm 0.2$ & $0.9 \pm 0.2$ & 0.186 \\
\hline Caton-Deschamps ratio ${ }^{a}$ & $1.0 \pm 0.2$ & $1.1 \pm 0.2$ & 0.132 \\
\hline Lateral patellofemoral angle ${ }^{\mathrm{a}}\left(^{\circ}\right)$ & $13.9 \pm 4.5$ & $12.3 \pm 4.3$ & 0.098 \\
\hline Trochlear dysplasia grade according to the Dejour classification ${ }^{\mathrm{b}}$ & & & 0.547 \\
\hline No & $56(94.9 \%)$ & $32(97.0 \%)$ & \\
\hline Type A & $3(5.1 \%)$ & $1(25.0 \%)$ & \\
\hline Kellgren-Lawrence grade ${ }^{b}$ & & & 0.637 \\
\hline Grade 1 & $0(0.0 \%)$ & $1(3.0 \%)$ & \\
\hline Grade 2 & $12(20.3 \%)$ & $7(21.2 \%)$ & \\
\hline Grade 3 & $37(62.7 \%)$ & $21(63.6 \%)$ & \\
\hline Grade 4 & $10(16.9 \%)$ & $4(12.1 \%)$ & \\
\hline Patellofemoral osteoarthritis stage according to Iwano's classification ${ }^{\text {b }}$ & & & 0.096 \\
\hline Stage 0 & $10(16.9 \%)$ & $12(36.4 \%)$ & \\
\hline Stage 1 & $27(45.8 \%)$ & $13(39.4 \%)$ & \\
\hline Stage 2 & $22(37.3 \%)$ & $8(24.2 \%)$ & \\
\hline Correction angle ${ }^{a}\left({ }^{\circ}\right)$ & $11.0 \pm 2.1$ & $11.3 \pm 2.6$ & 0.585 \\
\hline Time to hardware removal (months) ${ }^{a}$ & $21.7 \pm 6.2$ & $21.2 \pm 5.3$ & 0.664 \\
\hline Preoperative VAS score ${ }^{a}$ & $68.4 \pm 17.7$ & $63.4 \pm 19.8$ & 0.223 \\
\hline Preoperative IKDC subjective score ${ }^{a}$ & $33.8 \pm 11.8$ & $36.7 \pm 14.8$ & 0.308 \\
\hline Preoperative Kujala score ${ }^{a}$ & $38.3 \pm 14.7$ & $37.5 \pm 16.9$ & 0.812 \\
\hline
\end{tabular}

$B M I$ body mass index, VAS Visual analogue scale, IKDC International Knee Documentation Committee

${ }^{a}$ The values are given as the mean and standard deviation

$\mathrm{b}$ The values are given as the number of patients, with the percentage in parenthesis

dysplasia [27] were evaluated. In addition, the CatonDeschamps index and Blackburne-Peel ratio were used to assess patellar height and the lateral patellofemoral angle was used to measure patella tilt [28-30]. Two orthopedic surgeons who were not involved in the surgery measured all radiographic parameters with an interval of 6 weeks and were blinded to each other's measurements. Arthroscopic assessment was performed at the time of the initial operation and at the time of removal of the fixed plate. The total size of the cartilage lesion for each compartment of the knee was measured using 5-mm hook portion of an arthroscopic probe, and the severity of cartilage lesion was evaluated according to ICRS grading system [31] (Fig. 2a, b). All arthroscopic measurements were recorded immediately after the surgery by the orthopedic surgeon who performed the MOWHTO, and the assessments related to arthroscopic findings used in this study were based on this record.

\section{Statistical analysis}

All statistical analyses were conducted using IBM SPSS Statistics version 23.0 (IBM Corp., Released 2015, Armonk, NY, USA). A non-inferiority test was performed by examining whether the $95 \%$ confidence interval (CI) for the difference of Kujala score assessed at the time of second-look operation between the two groups 


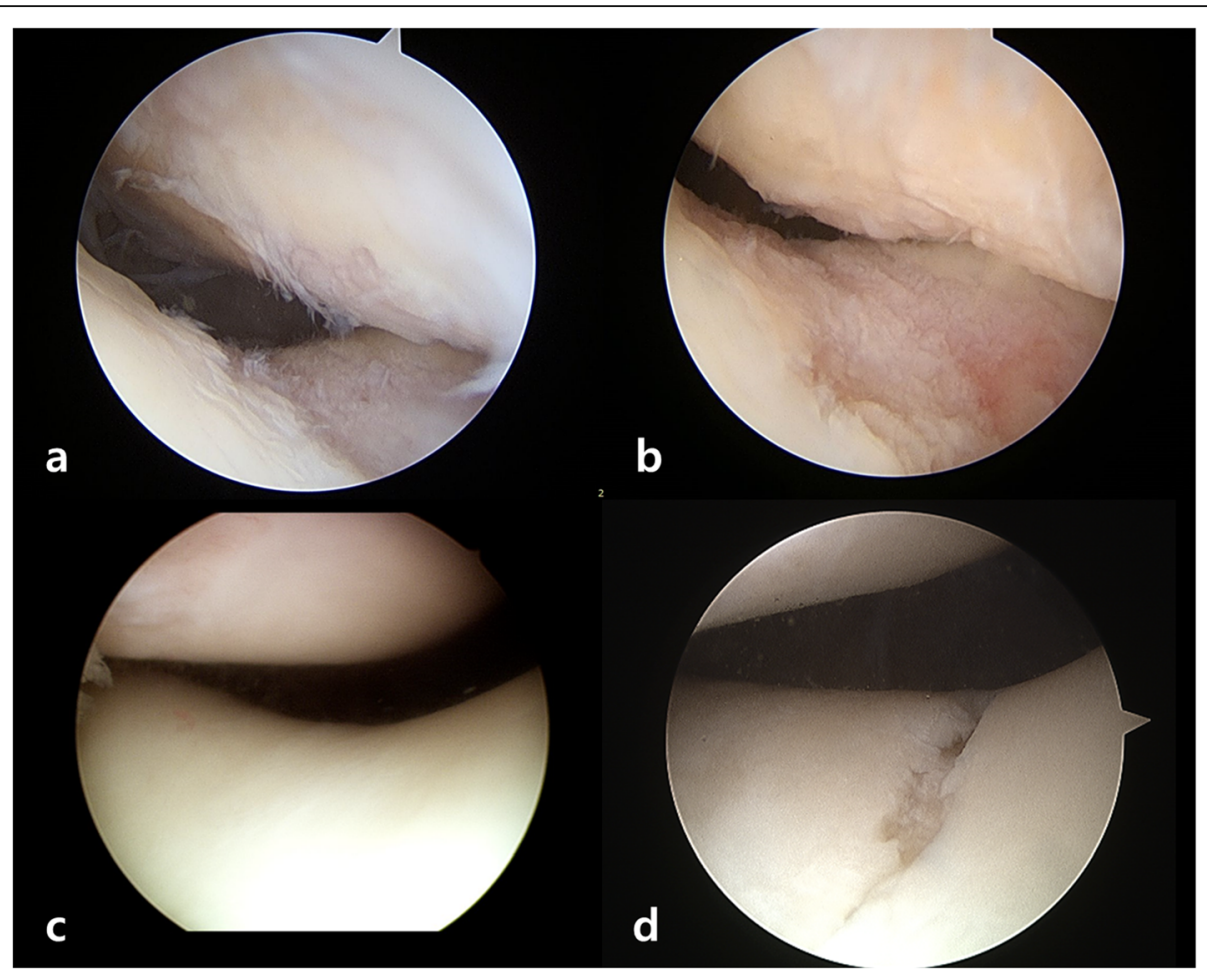

Fig. 2 Comparison of an arthroscopic assessment at initial operation and at the time of second-look operation. a Patient of group 1 showed preexisting cartilage lesions on patellofemoral joint (patella; ICRS grade III cartilage lesions with about 15x15mm size, trochlea; ICRS grade III cartilage lesions with about $5 \times 20 \mathrm{~mm}$ size), $\mathbf{b}$ which were observed to be progressed at second-look operation (patella; ICRS grade III cartilage lesions with about $15 \times 15 \mathrm{~mm}$ size, trochlea; ICRS grade III cartilage lesions with about 20x25mm size). c Patient of group 2 without any preexisting cartilage lesions on patellofemoral joint $\mathbf{d}$ showed development of cartilage lesions at second-look operation (trochlea; ICRS grade III cartilage lesions with about $3 \times 15 \mathrm{~mm}$ size). ICRS International Cartilage Repair Society

was less than the non-inferiority margin. The Kujala scale was set as a basis of comparison regarding clinical outcomes, since it is known as a valid and reliable scale for discriminating the differences in the severity of anterior knee pain [22]. The non-inferiority margin was set as 14, which is the reported value of minimal detectable change of the Kujala score [32]. In addition, the sample size was calculated on the basis of the abovementioned non-inferiority test. The reference value of the standard deviation (15.1) was adopted from a previous study [33]. By setting the significance level (alpha) at $5 \%$ and power (1-beta) at $90 \%$, a minimum of 20 patients in each group turned out to be adequate to detect the difference between the two groups.

Bivariate analysis was performed to compare the baseline characteristics, clinical outcomes, and radiographic outcomes between the groups. Student's t-test and Mann-Whitney U test were used for continuous variables, while Pearson's chi-squared and Fisher's exact tests were performed to evaluate categorical variables. To investigate the difference of change over time in the size of cartilage lesions between the two groups, repeated measures analysis of variance test was used. Wilcoxon-signed rank test was performed to compare the osteoarthritis grade of the patellofemoral joint and the grade of cartilage lesions at two separate time points. The kappa coefficient was used to evaluate the reliability for the evaluation of radiographic osteoarthritis grade [34], which revealed that inter-rater agreement regarding the radiographic osteoarthritis grade of the tibiofemoral joint and patellofemoral joint were 0.813 and 0.735 at preoperative time, respectively, and 0.781 and 0.761 at the time of second-look operation, respectively. A $P$ value $<0.05$ was considered statistically significant.

\section{Results}

Clinical results assessed using VAS, IKDC subjective, and Kujala scores showed overall improvement from baseline to the time of second-look operation, with no significant difference between the two groups at each time point (Fig. 3). The 95\% CI of the difference of Kujala score assessed at the time of second-look operation ranged from -7.88 to 7.56 , which did not exceed the non-inferiority margin of 14 points. Accordingly, clinical outcomes regarding anterior knee pain of group 1 was not statistically inferior to that of group 2 .

There were no significant differences between the two groups regarding radiographic factors assessed at the 


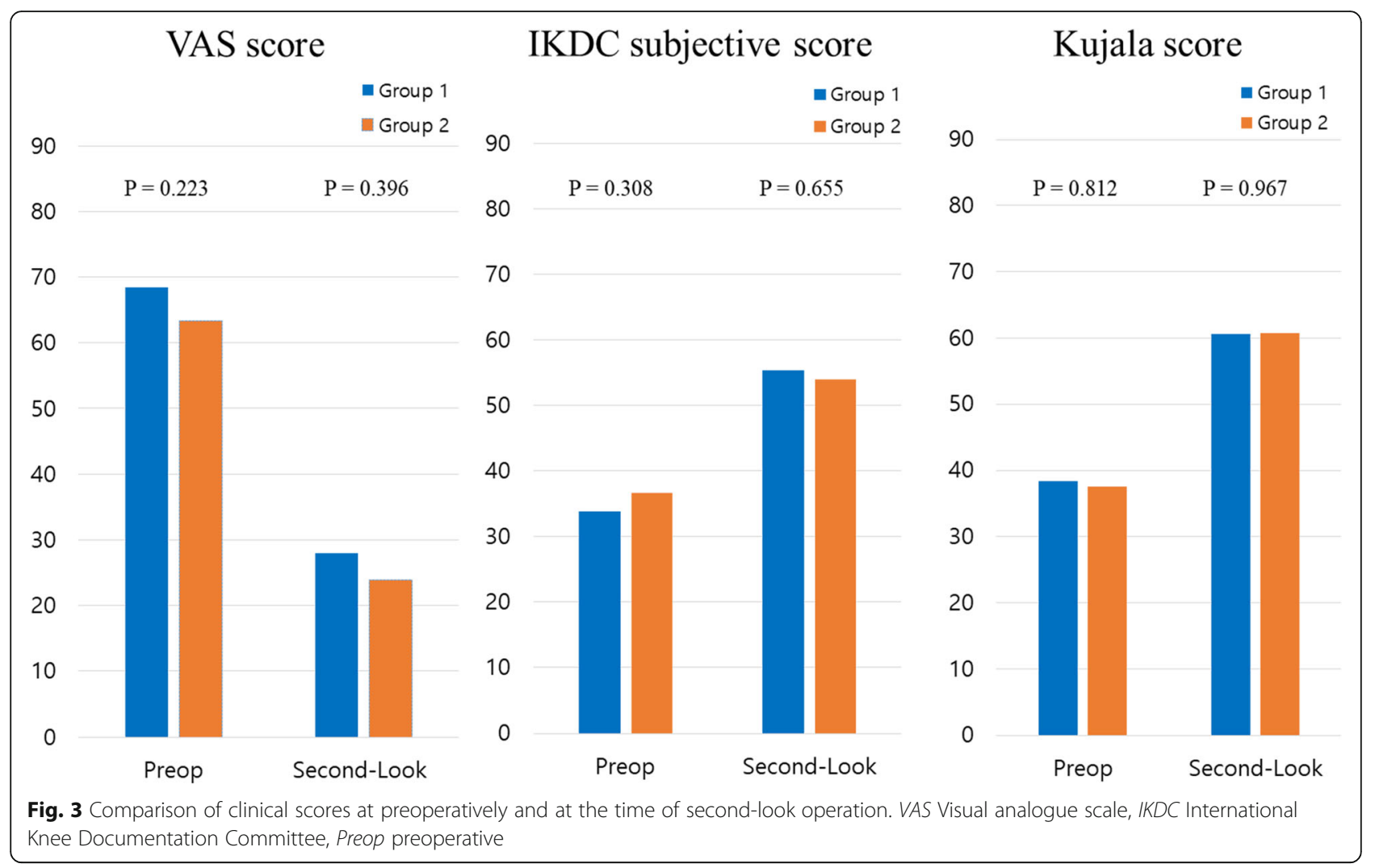

preoperative time as well as at the time of second-look operation (Tables 1,2). Comparing the osteoarthritis grade of the patellofemoral joint at second-look operation with the initial operation, both groups showed a tendency to progress, but without statistical significance (Table 3).

Comparison of the size and grade of cartilage lesions between the initial and second-look operations was analyzed. The size of cartilage lesion of the patellofemoral joint increased with time in both groups $(P=0.003)$. However, group-by-time interaction between the two groups was not statistically significant, indicating that the degree of change did not differ between the two groups (Fig. 4a). Consistently, there was no statistically significant difference in the frequency of progression of the cartilage lesion grade between the two groups (Table 4). Comparison of the cartilage lesion grade between two time points showed a tendency to deteriorate in both groups, but that of group 1 was not statistically significant whereas that of group 2 was statistically significant ( $P=0.071$ for group 1 and $P=0.007$ for group 2) (Table 5). In the medial compartment of the knee, the size of the cartilage lesions in both groups decreased over time $(P=0.000)$, whereas group-by-time interaction was not statistically significant (Fig. 4b). The macroscopic regeneration staging system of Koshino et al. was used to compare the grade of medial compartment cartilage lesion [35], which revealed that the frequency of cartilage regeneration was not statistically different between the two groups (Table 4).

\section{Discussion}

The principal finding of the current study was that MOWHTO contributes to the progression of osteoarthritis of the patellofemoral joint regardless of the preexisting cartilage status, but this was not considered to be directly associated with clinical outcome. In addition, the clinical outcome of MOWHTO in patients with preexisting cartilage lesions of the patellofemoral joint was not inferior to those with normal cartilage. This study could suggest that the preexisting focal cartilage lesions on the patellofemoral joint, less than ICRS grade 4, would not be a hindrance to perform MOWHTO.

MOWHTO has been known to negatively affect the patellofemoral joint as a result in the change of patellar position. Decreased patellar height and an altered patellofemoral alignment increases patellofemoral contact pressure [7-9], subsequently increasing the risk of osteoarthritis progression [36]. There are several preceding studies that performed an arthroscopic assessment of the progression of patellofemoral osteoarthritis resulting from MOWHTO [11-14, 37]. However, it is difficult to conclude that MOWHTO definitely contributes to the progression of patellofemoral osteoarthritis. Although 
Table 2 Comparison of radiographic parameters at the time of second-look operation

\begin{tabular}{|c|c|c|c|}
\hline Variable & $\begin{array}{l}\text { Group } 1 \\
(n=59)\end{array}$ & $\begin{array}{l}\text { Group } 2 \\
(n=33)\end{array}$ & $P$ value \\
\hline Load-bearing axis deviation ${ }^{\text {a }}(\%)$ & $64.3 \pm 9.2$ & $64.2 \pm 6.4$ & 0.983 \\
\hline Hip-Knee-Ankle angle ${ }^{a}$ (valgus, ${ }^{\circ}$ ) & $4.3 \pm 2.6$ & $4.0 \pm 1.7$ & 0.542 \\
\hline Medial proximal tibial angle ${ }^{\text {a }}\left({ }^{\circ}\right)$ & $93.7 \pm 3.0$ & $93.5 \pm 2.4$ & 0.807 \\
\hline Joint line convergence angle ${ }^{a}\left({ }^{\circ}\right)$ & $1.8 \pm 2.2$ & $1.7 \pm 2.0$ & 0.846 \\
\hline Posterior tibial slope $\left(^{\circ}\right)$ & $6.8 \pm 4.6$ & $7.7 \pm 4.0$ & 0.346 \\
\hline Blackburne-Peel ratio ${ }^{a}$ & $0.7 \pm 0.1$ & $0.8 \pm 0.2$ & 0.146 \\
\hline Caton-Deschamps ratio ${ }^{a}$ & $0.8 \pm 0.2$ & $0.9 \pm 0.2$ & 0.107 \\
\hline Lateral patellofemoral angle ${ }^{a}\left(^{\circ}\right)$ & $13.5 \pm 4.2$ & $12.3 \pm 5.6$ & 0.258 \\
\hline Kellgren-Lawrence grade ${ }^{b}$ & & & 0.441 \\
\hline Grade 1 & $0(0.0 \%)$ & $1(3.0 \%)$ & \\
\hline Grade 2 & $8(13.6 \%)$ & $7(21.2 \%)$ & \\
\hline Grade 3 & $43(72.9 \%)$ & $21(63.6 \%)$ & \\
\hline Grade 4 & $8(13.6 \%)$ & $4(12.1 \%)$ & \\
\hline $\begin{array}{l}\text { Patellofemoral osteoarthritis stage } \\
\text { according to Iwano's classification }\end{array}$ & & & 0.124 \\
\hline Stage 0 & $8(13.6 \%)$ & $10(30.3 \%)$ & \\
\hline Stage 1 & $25(42.4 \%)$ & $13(39.4 \%)$ & \\
\hline Stage 2 & 26 (44.1\%) & 10 (30.3\%) & \\
\hline
\end{tabular}

a The values are given as the mean and standard deviation

${ }^{b}$ The values are given as the number of patients, with the percentage in parenthesis

increased contact pressure of the patellofemoral joint may theoretically lead to progression of osteoarthritis in the affected joint, there are many variables to be considered. The progression of cartilage degeneration might be attributable to the normal age-dependent joint degeneration, as noted in preceding studies [12-14]. Moreover, preexisting cartilage lesions on the patellofemoral joint,

Table 3 Assessment of radiographic osteoarthritis grade of patellofemoral joint according to Iwano's classification system at second-look operation compared with the initial operation

\begin{tabular}{|c|c|c|c|}
\hline & $\begin{array}{l}\text { Group } 1 \\
(n=59)\end{array}$ & $\begin{array}{l}\text { Group } 2 \\
(n=33)\end{array}$ & $P$ value ${ }^{b}$ \\
\hline Preoperative $^{a}$ & & & 0.096 \\
\hline Stage 0 & $10(16.9 \%)$ & $12(36.4 \%)$ & \\
\hline Stage 1 & $27(45.8 \%)$ & $13(39.4 \%)$ & \\
\hline Stage 2 & $22(37.3 \%)$ & $8(24.2 \%)$ & \\
\hline Postoperative (Second-Look) a & & & 0.124 \\
\hline Stage 0 & $8(13.6 \%)$ & $10(30.3 \%)$ & \\
\hline Stage 1 & $25(42.4 \%)$ & $13(39.4 \%)$ & \\
\hline Stage 2 & $26(44.1 \%)$ & $10(30.3 \%)$ & \\
\hline$P$ value ${ }^{c}$ & 0.109 & 0.157 & \\
\hline
\end{tabular}

a The values are given as the number of patients, with the percentage in parenthesis

b Statistical significance was evaluated using Pearson's Chi-squared or Fisher's exact test

c Statistical significance was evaluated compared with the preoperative measurement values using Wilcoxon signed rank test which were frequently encountered during surgery, should be taken into account. Focal articular cartilage defects have been known to be a predisposing factor of osteoarthritis [16]. To determine whether MOWHTO affects the progression of patellofemoral osteoarthritis, the effect of articular cartilage status of the patellofemoral joint at the time of initial operation on the surgical outcomes should be clarified first.

Thus, the authors compared the surgical outcome of the two groups according to the cartilage status of the patellofemoral joint observed in the arthroscopic assessment performed during the initial operation. Although the two groups were classified according to the preexisting cartilage status, the proportion of the degree of patellofemoral joint osteoarthritis was not different between the two groups. This was observed not only in preoperative comparison but also at the time of second-look operation. However, osteoarthritis of the patellofemoral joint showed a tendency to progress in both groups, which was consistent with the arthroscopic assessment results. The size of cartilage lesions increased after MOWHTO in both groups, whereas the degree of change over time between the two groups was not different. The severity of cartilage lesions according to the ICRS grading system also seemed to deteriorate in both groups, but, interestingly, it was not statistically significant in the patients with preexisting cartilage lesion. It can be assumed that there was little room for arthritis progression in group 1, compared to group 2 . 


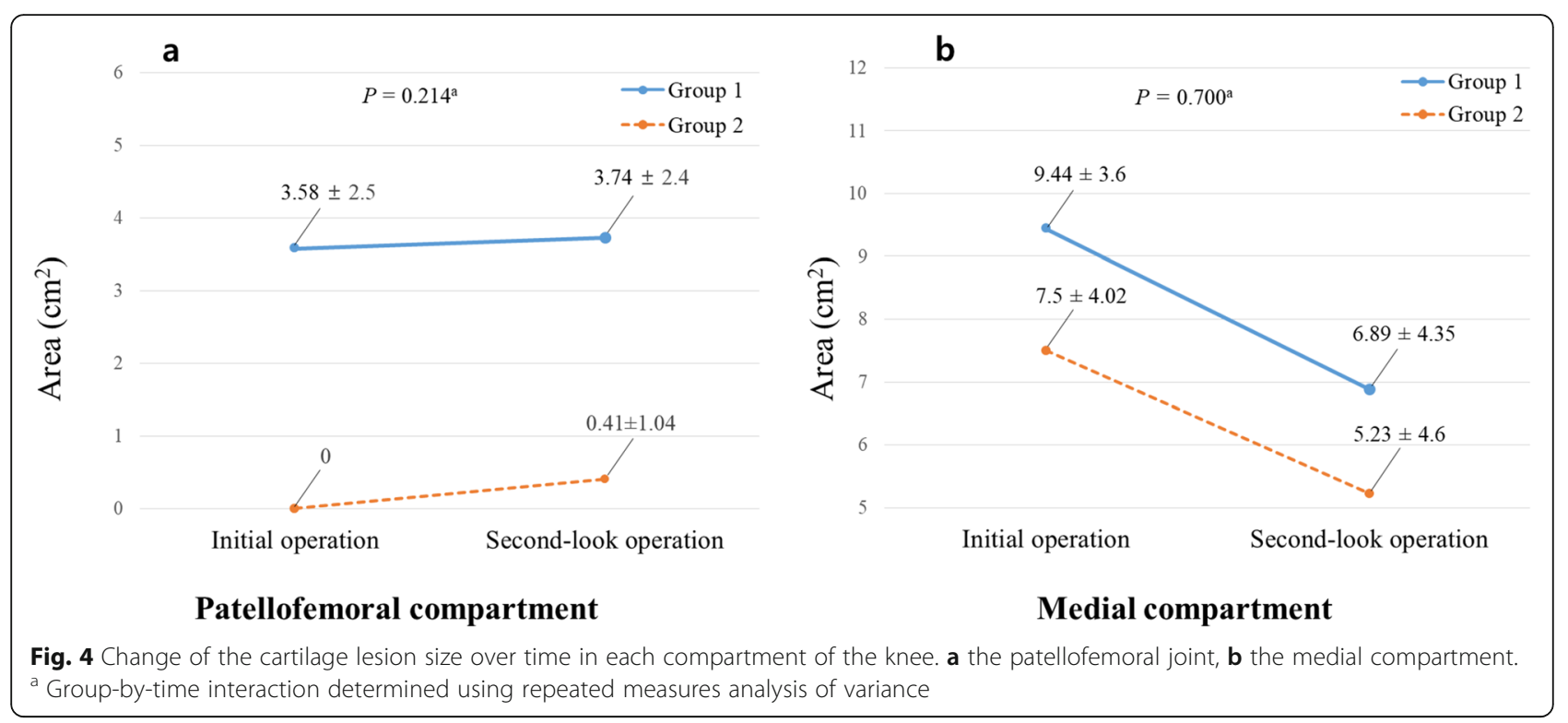

Taking into consideration the above-mentioned findings, MOWHTO could be considered to have an adverse effect on the patellofemoral joint regardless of the preexisting cartilage status.

However, apart from the results of the radiographic and arthroscopic measurements, clinical outcomes in both groups showed overall improvement. Moreover, there was no significant difference between the two groups in clinical outcomes with respect to anterior knee pain, which demonstrates that preexisting cartilage lesions of the patellofemoral joint would not be crucial factors affecting the surgical outcomes. Various reasons may explain the discrepancy between the objective assessments and clinical outcomes in the current study. First, we had already excluded patients with symptomatic anterior knee pain and those with radiographic evidence of severe osteoarthritis on the patellofemoral joint prior to surgery. Second,

Table 4 Assessment of the cartilage lesion grade at secondlook operation compared with the initial operation

\begin{tabular}{|c|c|c|c|}
\hline & $\begin{array}{l}\text { Group } 1 \\
(n=59)\end{array}$ & $\begin{array}{l}\text { Group } 2 \\
(n=33)\end{array}$ & $P$ value ${ }^{c}$ \\
\hline Medial compartment ${ }^{a}$ & & & 0.732 \\
\hline Stage A (No regeneration) & $18(30.5 \%)$ & $8(24.2 \%)$ & \\
\hline Stage B (Partial coverage) & $32(54.2 \%)$ & $18(54.5 \%)$ & \\
\hline Stage C (Total coverage) & $9(15.3 \%)$ & $7(21.2 \%)$ & \\
\hline Patellofemoral compartment ${ }^{b}$ & & & 0.136 \\
\hline Not progressed & $49(83.1 \%)$ & $23(69.7 \%)$ & \\
\hline Progressed & $10(16.9 \%)$ & $10(30.3 \%)$ & \\
\hline
\end{tabular}

${ }^{a}$ Based on macroscopic staging system of cartilage regeneration according to Koshino et al.

${ }^{\mathrm{b}}$ Based on International Cartilage Repair Society (ICRS) grading system

' Statistical significance was evaluated using Pearson's Chi-squared or Fisher's exact test the severity of preexisting cartilage lesions observed in the current study might be subtle. Although the stage of arthritis according to the Iwano classification showed a tendency to progress in both groups, severe osteoarthritis (Iwano classification stage 3 and 4) was not observed at the time of second-look operation. In addition, the source of anterior knee pain could be multifactorial including patella maltracking, malalignment of lower limb, and muscle imbalance [38]. Although osteoarthritis of the patellofemoral

Table 5 Assessment of cartilage lesion grade of patellofemoral joint according to ICRS grade system at second-look operation compared with the initial operation

\begin{tabular}{llll}
\hline & $\begin{array}{l}\text { Group 1 } \\
(n=59)\end{array}$ & $\begin{array}{l}\text { Group 2 } \\
(n=33)\end{array}$ & $P$ value ${ }^{\text {b }}$ \\
\hline Preoperative $^{\mathrm{a}}$ & $0(0.0 \%)$ & $20(60.6 \%)$ & \\
CRS grade 0 & $0(0.0 \%)$ & $13(39.4 \%)$ & \\
ICRS grade 1 & $12(20.3 \%)$ & $0(0.0 \%)$ & \\
ICRS grade 2 & $47(79.7 \%)$ & $0(0.0 \%)$ & \\
ICRS grade 3 & & & 0.000 \\
Postoperative (Second-Look) ${ }^{\text {a }}$ & & $16(48.5 \%)$ & \\
ICRS grade 0 & $0(0.0 \%)$ & $7(21.2 \%)$ & \\
ICRS grade 1 & $1(1.7 \%)$ & $5(15.2 \%)$ & \\
ICRS grade 2 & $5(8.5 \%)$ & $5(15.2 \%)$ & \\
ICRS grade 3 & $51(86.4 \%)$ & $0(0.0 \%)$ & \\
ICRS grade 4 & $2(3.4 \%)$ & 0.007 & \\
P value ${ }^{c}$ & 0.071 &
\end{tabular}

ICRS International Cartilage Repair Society

${ }^{a}$ The values are given as the number of patients, with the percentage in parenthesis

${ }^{\text {b }}$ Statistical significance was evaluated using Pearson's Chi-squared or Fisher's exact test

c Statistical significance was evaluated compared with the preoperative measurement values using Wilcoxon signed rank test 
joint may contribute to anterior knee pain, it may not be the only factor. Therefore, the clinical outcome could not be explained by the preexisting cartilage lesion alone.

There were recent studies to investigate relevant factors that influence the progression of patellofemoral osteoarthritis after MOWHTO. Yoon et al. addressed that overcorrection, a postoperative weight-bearing line ratio $>66.3 \%$, would lead to further progression of patellofemoral joint degeneration after MOWHTO [14]. Similarly, Tanaka et al. reported that cartilage lesions in the patellofemoral joint tended to progress after MOWHTO in patients with medial opening gap $\geq 13 \mathrm{~mm}$ or change in medial proximal tibial angle $\geq 9$ degrees [13]. Due to the methodological differences, there are limitations in applying and interpreting the results of previous studies in the present study. However, considering the mean value of the postoperative load-bearing axis in this study, the cohorts of the present study could be considered to be at risk for further progression of the patellofemoral osteoarthritis. Nevertheless, these variables did not differ between the two groups in this study. Considering that there were no significant differences in the progression of the patellofemoral osteoarthritis between the two groups despite being at the even condition at risk of the progress of patellofemoral degeneration, preexisting cartilage lesions on the patellofemoral joint would not be a major risk factor to lead to further progression of the patellofemoral osteoarthritis. Although the present study did not examine the factors that influence the progression of patellofemoral joint degeneration after MOWHTO, it could be suggested that preexisting cartilage lesions, which were considered a risk factor performing MOWHTO, would not be a hindrance to perform MOWHTO.

\section{Limitations}

The current study has several limitations. First, this study was based on a retrospective review, which could be associated with the risk of bias in evaluation. Second, the sample size is relatively small. However, sample size calculation indicated that the number of patients in each group was sufficient for comparison. Third, since this study was based on short-term results, it is too soon to generalize the results. Considering the increasing pattern of the cartilage lesion size of the patellofemoral joints with arthroscopic measurement in both groups, the mean follow-up period of the current study would not be sufficient to reflect the radiographic and clinical outcomes. Also, the possibility of normal age-dependent joint degeneration could not be completely excluded, since patients who did not undergo MOWHTO were not included as another control group in the current study, which was practically impossible. Fourth, data of the arthroscopic assessments were based on the medical records documented immediately after surgery, indicating that the intraoperative measurements could be associated with the risk of bias. However, since the evaluation with arthroscopic photos and movies are limited in measuring the cartilage lesion size as well as cartilage lesion depth, it would be appropriate to base it on consistently documented records which have been conducted blinded to this study. Fifth, inaccuracy in measuring the size of cartilage lesion under arthroscopic assessment should be taken into account. Although measuring the size of cartilage lesions was performed as precisely as possible, the accuracy of the measurement would still be limited since the articular surface did not have a flat contour and the border of the cartilage lesion was not clear in most of the cases. In addition, cartilage lesions of the patella and trochlea were not described separately. However, since the patellofemoral joint is a highly complicated structure consisting of a patella and femoral trochlea interacting with each other [39], the cartilage lesions of each compartment should not be evaluated individually. Therefore, the authors determined to present the result of the current study comprehensively combining both patella and trochlea lesions rather than describing them separately.

\section{Conclusions}

MOWHTO would contribute to the progression of osteoarthritis of the patellofemoral joint regardless of the preexisting cartilage status, without an association with clinical outcomes in short-term follow-up. Consequently, based on the comparative analysis results of the subjective and objective measures, it can be suggested that preexisting cartilage lesions on the patellofemoral joint are not crucial factors affecting the surgical outcomes.

\section{Supplementary information}

Supplementary information accompanies this paper at https://doi.org/10. 1186/s12891-019-2989-y

Additional file 1. Subgroup analysis of preoperative variables within group 1.

\begin{abstract}
Abbreviations
Cl: Confidence interval; ICRS: International Cartilage Repair Society; IKDC: International Knee Documentation Committee; MOWHTO: Medial open wedge high tibial osteotomy; VAS: Visual analog scale
\end{abstract}

\section{Acknowledgements \\ Not applicable.}

\section{Authors' contributions}

The project was coordinated by HS Moon and SH Kim. HS Moon drafted the manuscript, together with CH Choi, M Jung, SH Park, JK Shin. HS Moon and $\mathrm{SH}$ Kim generated the concept of the study. The design of the study, acquisition of data, analysis and interpretation of data were all done jointly by all authors. DY Lee and SH Kim revised the final draft critically for important intellectual content and approved the version to be submitted. All of authors agreed to be accountable for all aspects of the work in ensuring that questions related to the accuracy or integrity of any part of the work are appropriately investigated and resolved. 


\section{Funding}

Each author certifies that he or she has no commercial associations (eg, consultancies, stock ownership, equity interest, patent/licensing arrangements, etc) that might pose a conflict of interest in connection with the submitted article. There were no external sources of funding and none of the authors had any conflicts of interest.

\section{Availability of data and materials}

The datasets used and/or analyzed in this study available from the corresponding author on reasonable request.

\section{Ethics approval}

This study was ethically approved by the Institutional Review Board (IRB) of Gangnam severance hospital (ID Number:3-2018-0304).

\section{Consent for publication}

Not applicable.

\section{Competing interests}

The authors declare that they have no competing interests.

\section{Author details Hospital, Seoul, Republic of Korea. \\ Received: 2 July 2019 Accepted: 5 December 2019 \\ Published online: 14 December 2019}

${ }^{1}$ Arthroscopy and Joint Research Institute, Yonsei University College of Medicine, Seoul, Republic of Korea. ${ }^{2}$ Department of Orthopedic Surgery, Gangnam Severance Hospital, Yonsei University College of Medicine, Seoul, Republic of Korea. ${ }^{3}$ Department of Orthopedic Surgery, Severance Hospital, Yonsei University College of Medicine, Seoul, Republic of Korea. ${ }^{4}$ Department of Orthopedic Surgery, National Health Insurance Service Ilsan Hospital, Gyeonggi-do Republic of Korea. ${ }^{5}$ Department of Orthopedic Surgery, Saegil

\section{References}

1. Sprenger TR, Doerzbacher JF. Tibial osteotomy for the treatment of varus gonarthrosis. Survival and failure analysis to twenty-two years. J Bone Joint Surg Am. 2003;85(3):469-74.

2. Laprade RF, Spiridonov SI, Nystrom LM, Jansson KS. Prospective outcomes of young and middle-aged adults with medial compartment osteoarthritis treated with a proximal tibial opening wedge osteotomy. Arthroscopy. 2012;28(3):354-64.

3. Floerkemeier S, Staubli AE, Schroeter S, Goldhahn S, Lobenhoffer P. Outcome after high tibial open-wedge osteotomy: a retrospective evaluation of 533 patients. Knee Surg Sports Traumatol Arthroscopy. 2013; 21(1):170-80.

4. Lobenhoffer P, Agneskirchner JD. Improvements in surgical technique of valgus high tibial osteotomy. Knee Surg Sports Traumatol Arthroscopy. 2003;11(3):132-8.

5. Cotic M, Vogt $S$, Feucht MJ, Saier T, Minzlaff $P$, Hinterwimmer S, Imhoff AB Prospective evaluation of a new plate fixator for valgus-producing medial open-wedge high tibial osteotomy. Knee Surg Sports Traumatol Arthroscopy. 2015;23(12):3707-16.

6. Blackman AJ, Krych AJ, Engasser WM, Levy BA, Stuart MJ. Does proximal tibial osteotomy with a novel osteotomy system obtain coronal plane correction without affecting tibial slope and patellar height? Knee Surg Sports Traumatol Arthroscopy. 2015;23(12):3487-93.

7. Javidan P, Adamson GJ, Miller JR, Durand P Jr, Dawson PA, Pink MM, Lee $T Q$. The effect of medial opening wedge proximal tibial osteotomy on patellofemoral contact. Am J Sports Med. 2013;41(1):80-6.

8. Yang JH, Lee $\mathrm{SH}$, Nathawat $\mathrm{KS}$, Jeon $\mathrm{SH}$, Oh KJ. The effect of biplane medial opening wedge high tibial osteotomy on patellofemoral joint indices. Knee. 2013:20(2):128-32.

9. Bin SI, Kim HJ, Ahn HS, Rim DS, Lee DH. Changes in patellar height after opening wedge and closing wedge high Tibial osteotomy: a meta-analysis. Arthroscopy. 2016;32(11):2393-400.

10. Cahue S, Dunlop D, Hayes K, Song J, Torres L, Sharma L. Varus-valgus alignment in the progression of patellofemoral osteoarthritis. Arthritis Rheum. 2004;50(7):2184-90.

11. Kim KI, Kim DK, Song SJ, Lee SH, Bae DK. Medial open-wedge high Tibial osteotomy may adversely affect the Patellofemoral joint. Arthroscopy. 2017; 33(4):811-6.
12. Goshima K, Sawaguchi T, Shigemoto K, Iwai S, Nakanishi A, Ueoka K Patellofemoral osteoarthritis progression and alignment changes after openwedge high Tibial osteotomy do not affect clinical outcomes at mid-term follow-up. Arthroscopy. 2017;33(10):1832-9.

13. Tanaka T, Matsushita T, Miyaji N, Ibaraki K, Nishida K, Oka S, Araki D, Kanzaki $\mathrm{N}$, Hoshino Y, Matsumoto T, et al. Deterioration of patellofemoral cartilage status after medial open-wedge high tibial osteotomy. Knee Surg Sports Traumatol Arthroscopy. 2019;27(4):1347-54.

14. Yoon TH, Choi CH, Kim SJ, Kim SH, Kim NH, Jung M. Effect of medial openwedge high Tibial osteotomy on the Patellofemoral joint according to postoperative realignment. Am J Sports Med. 2019;47(8):1863-73.

15. LaPrade RF. Editorial commentary: is it the osteotomy that is causing the development of Patellofemoral osteoarthritis or is it the Normal progression of preexisting osteoarthritis? Arthroscopy. 2017;33(4):817-8.

16. Guettler JH, Demetropoulos CK, Yang KH, Jurist KA. Osteochondral defects in the human knee: influence of defect size on cartilage rim stress and load redistribution to surrounding cartilage. Am J Sports Med. 2004;32(6):1451-8.

17. Iwano T, Kurosawa H, Tokuyama H, Hoshikawa Y. Roentgenographic and clinical findings of patellofemoral osteoarthrosis. With special reference to its relationship to femorotibial osteoarthrosis and etiologic factors. Clin Orthopaedics Related Res. 1990;252:190-7.

18. Miniaci A, Ballmer FT, Ballmer PM, Jakob RP. Proximal tibial osteotomy. A new fixation device. Clin Orthopaedics Related Res. 1989;246:250-9.

19. Fujisawa $Y$, Masuhara $K$, Shiomi $S$. The effect of high tibial osteotomy on osteoarthritis of the knee. An arthroscopic study of 54 knee joints. Orthopedic Clinics North America. 1979;10(3):585-608.

20. Flandry F, Hunt JP, Terry GC, Hughston JC. Analysis of subjective knee complaints using visual analog scales. Am J Sports Med. 1991;19(2):112-8.

21. Irrgang JJ, Anderson AF, Boland AL, Harner CD, Kurosaka M, Neyret P, Richmond JC, Shelborne KD. Development and validation of the international knee documentation committee subjective knee form. Am J Sports Med. 2001;29(5):600-13.

22. Kujala UM, Jaakkola LH, Koskinen SK, Taimela S, Hurme M, Nelimarkka O. Scoring of patellofemoral disorders. Arthroscopy. 1993;9(2):159-63.

23. Kellgren JH, Lawrence JS. Radiological assessment of osteo-arthrosis. Ann Rheum Dis. 1957;16(4):494-502.

24. Iseki Y, Takahashi T, Takeda H, Tsuboi I, Imai H, Mashima N, Watanabe S, Yamamoto $\mathrm{H}$. Defining the load bearing axis of the lower extremity obtained from anterior-posterior digital radiographs of the whole limb in stance. Osteoarthr Cartil. 2009;17(5):586-91.

25. Paley D, Herzenberg JE, Tetsworth K, McKie J, Bhave A. Deformity planning for frontal and sagittal plane corrective osteotomies. Orthopedic Clin North America. 1994;25(3):425-65.

26. Hohmann E, Bryant $A$, Reaburn P, Tetsworth $K$. Is there a correlation between posterior tibial slope and non-contact anterior cruciate ligament injuries? Knee Surg Sports traumatol Arthroscopy. 2011;19(Suppl 1):S109-14.

27. Dejour D, Le Coultre B. Osteotomies in patello-femoral instabilities. Sports Med Arthrosc Rev. 2007;15(1):39-46.

28. Blackburne JS, Peel TE. A new method of measuring patellar height. J Bone Joint Surg Br Vol. 1977;59(2):241-2.

29. Caton J. Method of measuring the height of the patella. Acta Orthop Belg. 1989;55(3):385-6.

30. Laurin CA, Levesque HP, Dussault R, Labelle H, Peides JP. The abnormal lateral patellofemoral angle: a diagnostic roentgenographic sign of recurrent patellar subluxation. J Bone Joint Surg Am. 1978;60(1):55-60.

31. Mainil-Varlet P, Aigner T, Brittberg M, Bullough P, Hollander A, Hunziker E, Kandel $R$, Nehrer S, Pritzker K, Roberts S, et al. Histological assessment of cartilage repair: a report by the histology endpoint Committee of the International Cartilage Repair Society (ICRS). J Bone Joint Surg Am. 2003;85-A(Suppl 2):45-57.

32. Watson CJ, Propps M, Ratner J, Zeigler DL, Horton P, Smith SS. Reliability and responsiveness of the lower extremity functional scale and the anterior knee pain scale in patients with anterior knee pain. J Orthopaedic Sports Physical Therapy. 2005;35(3):136-46.

33. Lee YS, Lee SB, Oh WS, Kwon YE, Lee BK. Changes in patellofemoral alignment do not cause clinical impact after open-wedge high tibial osteotomy. Knee Surg Sports Traumatol Arthroscopy. 2016;24(1):129-33.

34. Landis JR, Koch GG. The measurement of observer agreement for categorical data. Biometrics. 1977:33(1):159-74.

35. Koshino T, Wada S, Ara Y, Saito T. Regeneration of degenerated articular cartilage after high tibial valgus osteotomy for medial compartmental osteoarthritis of the knee. Knee. 2003;10(3):229-36. 
36. Song $\mathrm{H}_{\text {, Song }} \mathrm{EK}$, Seo HY, Lee KB, Yim JH, Seon JK. Patellofemoral alignment and anterior knee pain after closing- and opening-wedge valgus high tibial osteotomy. Arthroscopy. 2012;28(8):1087-93.

37. Horikawa T, Kubota K, Hara S, Akasaki Y. Distal tuberosity osteotomy in open-wedge high tibial osteotomy does not exacerbate patellofemoral osteoarthritis on arthroscopic evaluation. Knee Surg Sports Traumatol Arthroscopy. 2019. https://doi.org/10.1007/s00167-019-05596-y.

38. Petersen W, Ellermann A, Gosele-Koppenburg A, Best R, Rembitzki IV, Bruggemann GP, Liebau C. Patellofemoral pain syndrome. Knee Surg Sports Traumatol Arthroscopy. 2014;22(10):2264-74.

39. Tecklenburg K, Dejour D, Hoser C, Fink C. Bony and cartilaginous anatomy of the patellofemoral joint. Knee Surg Sports Traumatol Arthroscopy. 2006; 14(3):235-40.

\section{Publisher's Note}

Springer Nature remains neutral with regard to jurisdictional claims in published maps and institutional affiliations.

Ready to submit your research? Choose BMC and benefit from:

- fast, convenient online submission

- thorough peer review by experienced researchers in your field

- rapid publication on acceptance

- support for research data, including large and complex data types

- gold Open Access which fosters wider collaboration and increased citations

- maximum visibility for your research: over $100 \mathrm{M}$ website views per year

At BMC, research is always in progress.

Learn more biomedcentral.com/submissions 